\title{
Two-Dimensional Spectral Estimation: A Radon Transform Approach
}

\author{
N. SRINIVASA, STUDENT MEMBER, IEEE, K. R. RAMAKRISHNAN, MEMBER, IEEE, K. RAJGOPAL, MEMBER, IEEE
}

(Invited Paper)

\begin{abstract}
A new technique for two-dimensional (2-D) spectral estimation of a stationary random field (SRF) is investigated in this paper. This is based on the extension of the Radon transform theory to stationary random fields (SRF's), proposed by Jain and Ansari [19]. Using the Radon transform, the 2-D estimation problem is reduced to a set of onedimensional (1-D) independent problems, which could then be solved using 1-D linear prediction (LP) or by any other high-resolution estimation procedure. This is unlike previous methods which obtain the 2-D power spectral density (PSD) estimate by using 1-D high-resolution techniques in the spirit of a separable estimator [2]. Examples are provided to illustrate the performance of the new technique. Various features of this approach are highlighted.
\end{abstract}

\section{INTRODUCTION}

$I^{\mathrm{N}}$ many applications of two-dimensional (2-D) signal processing such as sonar, radar, geophysics, and radio astronomy, the problem of estimation of the power spectral density (PSD) of a sampled stationary random field (SRF) from a finite set of observations is often encountered. The classical method of estimation of PSD using the periodogram results in poor resolution. A recent approach to obtain a highresolution estimate is to postulate a finite parametric model for the PSD. The observations are used to obtain the model parameters by an appropriate estimate procedure. In one dimension, the modeling techniques employing linear prediction (LP) theory have been widely investigated and are successfully being used to obtain the PSD estimate especially at a high signal-to-noise ratio (SNR) [1]. An extension to the 2-D case is the use of one-dimensional (1-D) LP models along each of the dimensions. A class of 2-D PSD estimators, known as the separable spectral estimators, employs 1-D estimation techniques sequentially along each of the dimensions [2]-[7]. Since it is crucial that the phase not be discarded at the intermediate step, in [3] and [4] discrete Fourier transform (DFT) is used along the first dimension and a high-resolution 1-D autoregressive (AR) spectral estimator along the other dimension. The resolution obtained along the first dimension is restricted by the DFT. Higher resolution along the first dimension is obtained by artificially extending the data while preserving the phase as well. The techniques used for generating additional data include the use of 1-D AR models [5], [6] and band-limited extrapolation [7]. The techniques used in [3]-[7], the 2-D high-resolution PSD was obtained by a separable operation, i.e., row-by-row operation followed by

Manuscript received April 16, 1986; revised August 29, and October 30, 1986.

The authors are with the Department of Electrical Engineering, Indian Institute of Science, Bangalore 560 012, India.

IEEE Log Number 8714602. column-by-column operation. In the presence of noise, as is the case in all applications, this separable class of estimation is not justified [7]-[9]. Further, separable estimation ignores the correlation between rows and columns. The interdimensional correlation is exploited by modeling the 2-D sequence using 2-D LP models [8]-[14]. However, in 2-D modeling, the choice of the model and the associated predictor filter mask, the appropriate order for the model, the order of computation of the model parameters, and the resulting characteristics of the spectral estimate are the major issues [2], [13]-[16]. For a review of various other classes of multidimensional spectral estimators, the reader is referred to [2].

This paper presents a novel approach for the 2-D PSD estimation from a finite set of observations of a 2-D SRF using the Radon transform approach. The basic 2-D estimation problem is converted into a set of 1-D independent problems using the central-slice theorem for stationary random fields [19]. The central-slice theorem relates the PSD of 1-D projections of an SRF and its 2-D PSD. The projections of SRF are estimated from available observations and the theorem is then applied to obtain the 2-D PSD.

The organization of the paper is as follows. Section II briefly reviews the theory of Radon transform of stationary random fields. Section III outlines the procedure for obtaining the 2-D PSD of an SRF from the observations, and also highlights the various features of this approach. Simulation results are presented, along with comments, illustrating the performance of the proposed technique in Section IV. Section $V$ concludes the paper.

\section{Radon Transform of Random Fields}

The Radon transform is the mathematical basis of Computer Tomography [17], [18]. Here, the basic data are a set of 1-D projections of a 2-D object obtained by integrating the object function along a set of lines. The projection process is called the 2-D Radon transforms. For a 2-D deterministic function $f(x, y)$ the Radon transform $\mathbb{R}$ is defined as

$$
\begin{aligned}
p_{\theta}(t) & =\mathbb{R}[f]=\int_{A B} f(x, y) d s \\
& =\int_{-\infty}^{\infty} \int_{\infty} f(x, y) \delta(x \cos \theta+y \sin \theta-t) d x d y
\end{aligned}
$$

where $d s$ is the elemental distance on the line $A B$ represented by the equation $x \cos \theta+y \sin \theta=t$, see Fig. 1 . It is to be noted that $p_{\theta}(t)$, the projection of the 2-D function $f(x, y)$ at an angle $\theta$ is a 1-D function in $t$. 


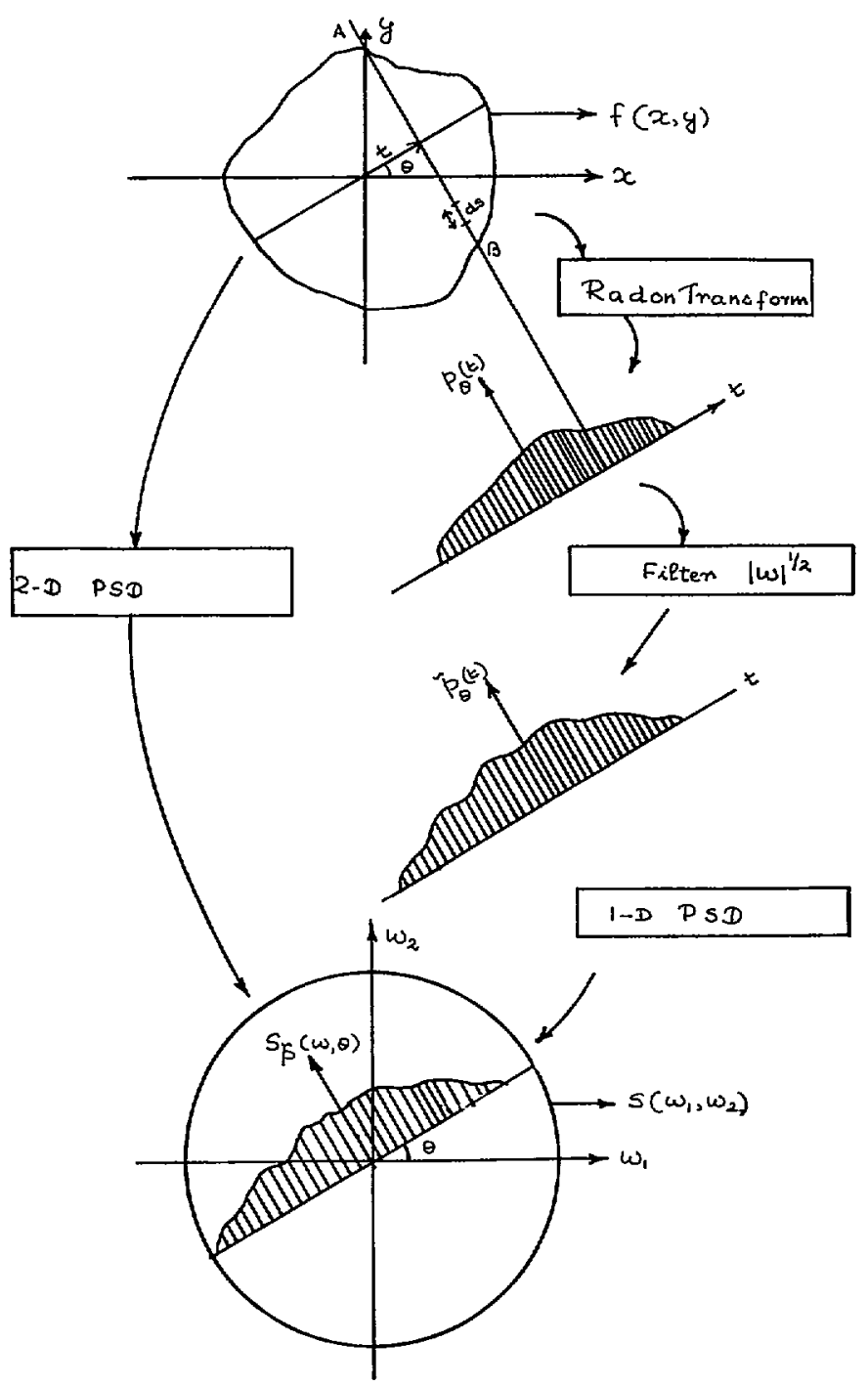

Fig. 1. The central-slice theorem for stationary random fields.

\section{Central-Slice Theorem}

A fundamental result, on which various techniques for reconstructing multidimensional signals from their projections rely, is the central-slice theorem or the projection-slice theorem [18]. It states that the $(N-1)$-dimensional Fourier transform of a projection is a slice through the $N$-dimensional Fourier transform of the original function itself. For the sake of simplicity, consider the 2-D case.

Let $S\left(\omega_{1}, \omega_{2}\right)$ be the 2-D Fourier transform of the function $f(x, y)$, in Cartesian coordinates:

$$
S\left(\omega_{1}, \omega_{2}\right)=\int_{-\infty}^{\infty} \int(x, y) \exp \left(-j 2 \pi\left(\omega_{1} x+\omega_{2} y\right)\right) d x d y .
$$

Let $P_{\theta}(\omega)$ denote the 1-D Fourier transform of the projection $p_{\theta}(t)$ :

$$
P_{\theta}(\omega)=\int_{-\infty}^{\infty} p_{\theta}(t) \exp (-j 2 \pi \omega t) d t
$$

If $S(\omega, \theta)$ denotes the value of the Fourier transform of $f(x, y)$ in polar coordinates, then

$$
S(\omega, \theta)=P_{\theta}(\omega)
$$

As a direct consequence of this theorem, it is possible to fill up the complete Fourier plane by taking the Fourier transform of projections at various angles.

As the line integral of an SRF does not exist in the meansquare sense, the $R$ operator is replaced by a modified operator $\widetilde{\mathcal{Q}}$ which is the Radon transform $R$ followed by a 1-D convolution operator $H$ whose frequency response is $|\omega|^{1 / 2}$ [19]. The resulting function $\tilde{p}_{\theta}(t)$ is given by

$$
\tilde{p_{\theta}}(t)=\tilde{\mathbb{R}}[f]=R[f] * H=p_{\theta}(t) * H
$$

and using (4), we obtain

$$
\tilde{p}_{\theta}(t)=\int_{-\infty}^{\infty}|\omega|^{1 / 2} S(\omega, \theta) \exp (+j 2 \pi \omega t) d \omega .
$$

Let the 1-D PSD of $\tilde{p}_{\theta}(t)$ be denoted by $S_{\tilde{p}}(\omega, \theta)$. According to the modified central-slice theorem, the 1-D PSD of $\tilde{p}_{\theta}(t)$ of an SRF $f(x, y)$ is the central-slice at an angle $\theta$ of the 2-D PSD $S\left(\omega_{1}, \omega_{2}\right)$ of $f(x, y)$. In otherwords:

$$
S(\omega \cos \theta, \omega \sin \theta)=S_{p}(\omega, \theta)
$$

See Fig. 1.

In applications wherein projections of an SRF are available directly, the computation of the 2-D PSD is rather straightforward. In cases where only the observations are available instead of the projections, the need of estimating the projections from these observations arises. Starting from the observation set, the procedure of estimating the 2-D PSD and the various features of this approach are dealt with in the next section.

\section{Two-Dimensional Spectral Estimation}

The basic idea here is to use the Radon transform to reduce the 2-D sequence to a set of 1-D sequences. The 1-D problem can then be solved by using 1-D LP theory or by any other estimation procedure.

We assume the discrete observations $f\left(n_{1}, n_{2}\right)$ where $n_{1}=$ $1,2, \cdots, N_{1}$ and $n_{2}=1,2, \cdots, N_{2}$ are obtained by sampling the continuous SRF at the Nyquist rate. Due to the discreteness of the available data, it is not possible to obtain the projections directly using (1). Without taking recourse to the discrete Radon transform which invariably involves interpolation of the 2-D observations, an alternative approach of approximating the integral in (1) is made by making the following assumptions. The value of each data point is assumed to be a constant over a square element of unit area. This is analogous to the "pixel" assumption in digital image processing. Further, the integral in (1) is replaced by a summation operator.

Let $p_{\theta_{i}}\left(t_{j}\right)$ denote the integral (summation in the discrete case) of $f\left(n_{1}, n_{2}\right)$ along the line parameterized by the discrete variables $\theta_{i}$ and $t_{j}$. For the sake of simplicity, we retain only 


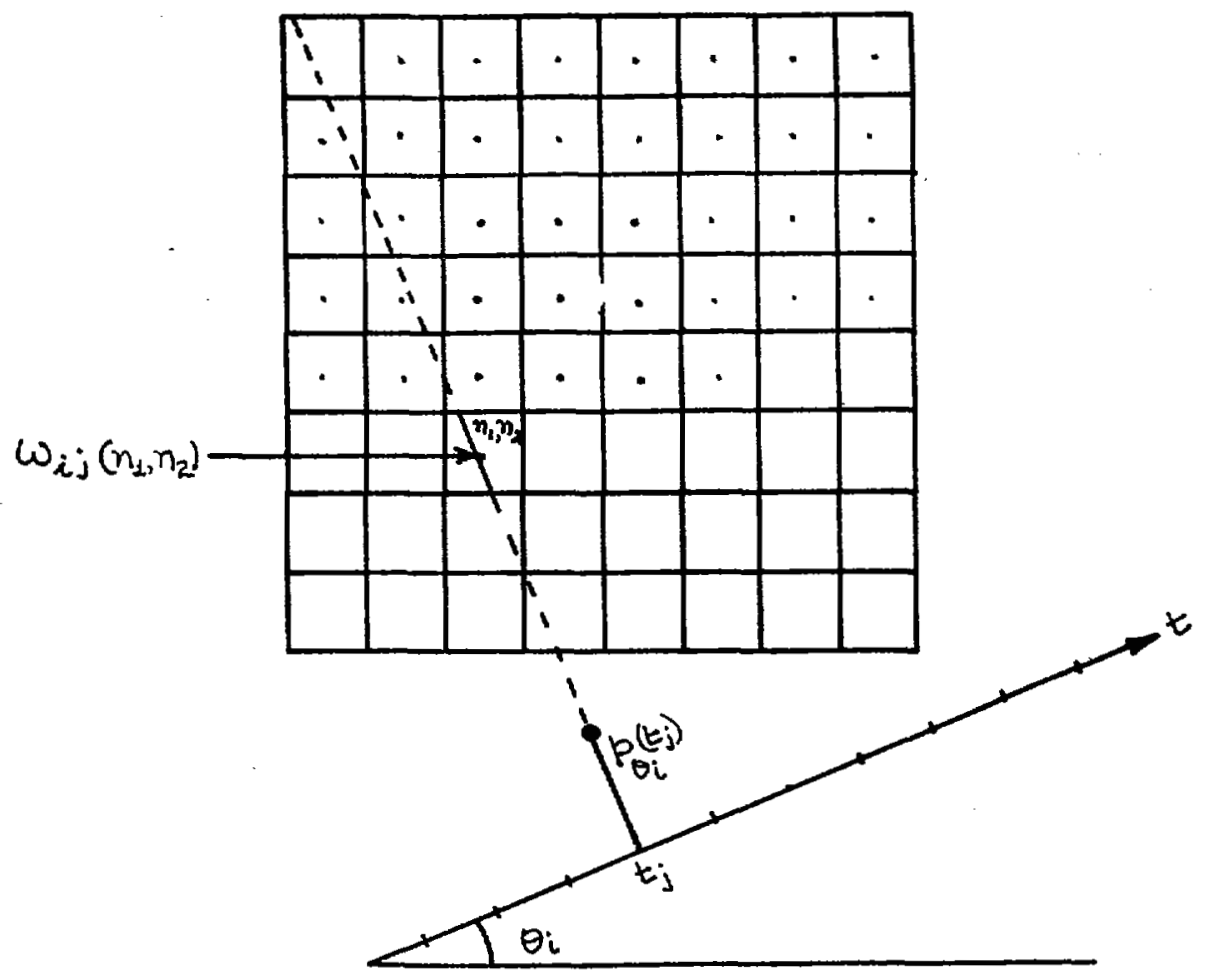

Fig. 2. Computation of $w$ 's for obtaining projection $p(j)$ from the geometry.

the indices $i$ and $j$ in subsequent discussions:

$$
p_{i}(j)=\sum_{n_{1}=1}^{N_{1}} \sum_{n_{2}=1}^{N_{2}} w_{i j}\left(n_{1}, n_{2}\right) f\left(n_{1}, n_{2}\right)
$$

where $w_{i j}\left(n_{1}, n_{2}\right)$ is a weighting factor determined by some geometrical considerations [20]. For example, $w_{i j}\left(n_{1}, n_{2}\right)$ can be made proportional to the length of intersection of the $j$ th ray in the $i$ th projection with the square element $\left(n_{1}, n_{2}\right)$, see Fig. 2. A simpler scheme is to use $w_{i j}\left(n_{1}, n_{2}\right)=0$ or 1 according to whether the ray on the square element $\left(n_{1}, n_{2}\right)$ intersects a circle of a given radius that is centered on the $\left(n_{1}, n_{2}\right)$ element. For small values of $N_{1}$ and $N_{2}, w_{i j}\left(n_{1}, n_{2}\right)$ 's can be precomputed and stored, so that the evaluation of $p_{i}(j)$ 's will become faster. For large values of $N_{1}$ and $N_{2}$ some symmetry conditions offered by the equispaced sampling along the two dimensions can be exploited for faster computation of $w_{i j}$ 's. Thus $p_{i}(j)$ is equal to the weighted sum of the observations associated with the respective ray. Each discrete projection $p_{i}(\cdot)$ is now filtered by a 1-D filter whose frequency response is $|\omega|{ }^{1 / 2}$. The fast convolution method can be made use of for this purpose. The resultant sequence $\tilde{p}_{i}(\cdot)$ is now used to compute an estimate of $S\left(\omega_{k}, \theta_{i}\right)$ which is the discrete counterpart of $S(\omega, \theta)$. For example, $\tilde{p}_{i}(\cdot)$ is modeled with 1-D AR or autoregressive moving average (ARMA) models. The corresponding slices of $S\left(\omega_{k}, \theta_{i}\right)$ is approximated by $\tilde{S}\left(\omega_{k}, \theta_{i}\right)$ which can be computed from the model parameters. This procedure is repeated for various other angles. By making use of the property

$$
p_{\theta}(t)=p_{\theta+\pi}(-t)
$$

only projections spanning the range of $\left[0^{\circ}, 180^{\circ}\right)$ need to be computed. The procedure for obtaining the 2-D PSD from the observations can be summarized in the following steps.

1) Obtain the discrete projection $p_{i}(\cdot)$ from the observations $f\left(n_{1}, n_{2}\right)$ at a particular angle $\theta_{i}$ using (7).

2) Filter the projection $p_{i}(\cdot)$ by a 1-D filter whose frequency response is $|\omega|^{1 / 2}$ to obtain $\tilde{p}_{i}(\cdot)$.

3) Model the filtered projection by an appropriate 1-D linear prediction model and compute the corresponding 1-D LP spectrum from the model parameters. Alternatively, any other 1-D high-resolution spectral estimation technique can be used. The resulting 1-D PSD forms a slice of the 2-D PSD along $\theta_{i}$.

4) Repeat steps 1 to 3 for various angles $\left[0^{\circ}, 180^{\circ}\right.$ ) to obtain the complete 2-D PSD estimate.

Since each of the slices of the 2-D PSD are computed independently by $1-D$ modeling techniques it is possible to tailor the 2-D PSD by choosing different models for different slices. This feature allows the user to approximate the various slices at different accuracy. Another interesting feature in this approach is that it is possible to obtain the estimate over any angular region of the 2-D PSD. By obtaining projections at evenly spaced angles spanning $\left[0^{\circ}, 180^{\circ}\right)$ and using the same distance between any two consecutive rays in a projection, the 2-D PSD is obtained on a polar grid (Fig. 3). It should be noted that the resulting polar raster has a greater number of samples near the origin than farther away. Thus the PSD estimate obtained in this method has a nonuniform resolution. If any area of the PSD is required at a higher resolution, complex demodulation of signals can be used to steer the region of interest towards the origin. 


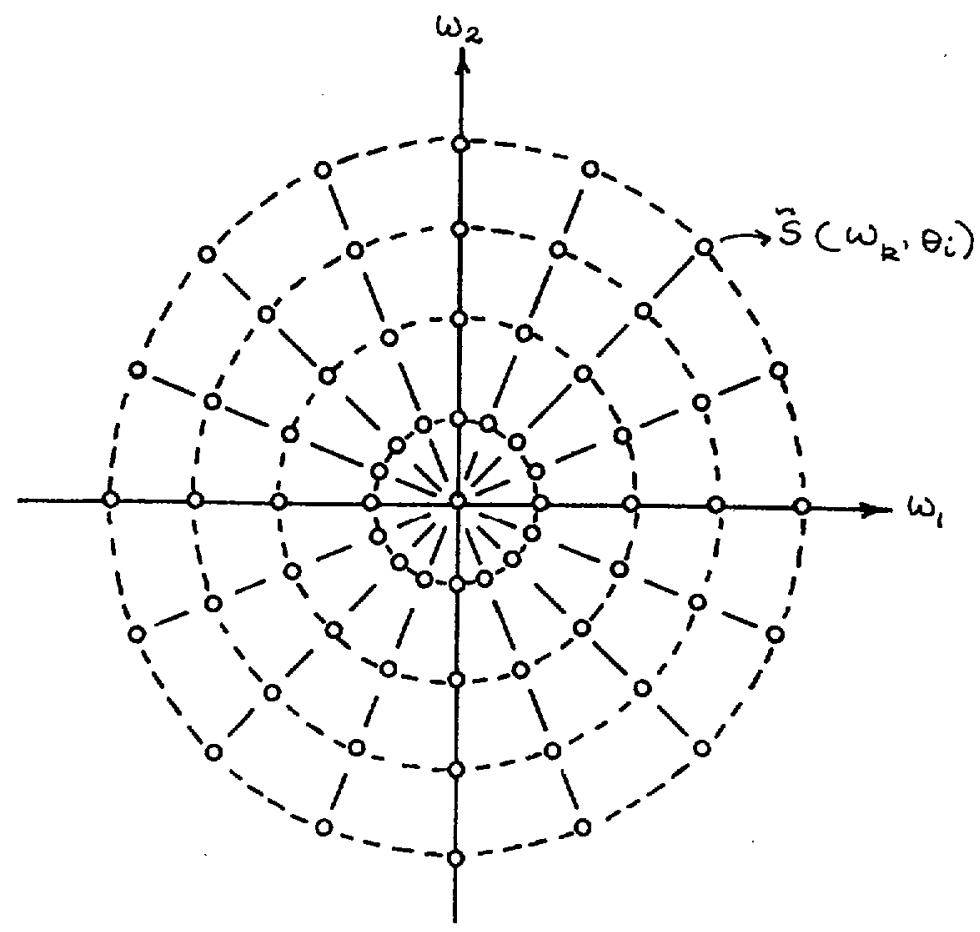

Fig. 3. Slices of the 2-D PSD on a polar raster.

The present trend in real-time signal processing is to devise algorithms for implementation on parallel machines. As this approach reduces the 2-D problem into a set of 1-D independent problems which can be processed concurrently, it is highly amenable for parallel processing.

\section{SiMULATION RESULTS}

As an application of the proposed technique, we consider the problem of resolving two sinusoids in white noise. We present two examples where the 2-D sequence $f\left(n_{1}, n_{2}\right)$ is composed of two equiamplitude 2-D sinusoids and Gaussian white noise sequence $g\left(n_{1}, n_{2}\right)$ of zero mean and variance $\sigma_{g}^{2}$.

A. Example 1

$$
\begin{aligned}
f\left(n_{1}, n_{2}\right)= & \cos \left(0.4688 \pi n_{1}\right) \cos \left(0.5313 \pi n_{2}\right) \\
& +\cos \left(0.5938 \pi n_{1}\right) \cos \left(0.6563 \pi n_{2}\right) \\
& +g\left(n_{1}, n_{2}\right)
\end{aligned}
$$

for

$$
\begin{aligned}
& 1 \leq n_{1} \leq N_{1} \\
& 1 \leq n_{2} \leq N_{2}
\end{aligned}
$$

and

$$
\begin{gathered}
N_{1}=N_{2}=32 \\
\sigma_{g}^{2}=0.025 \\
\text { SNR }=0 \mathrm{~dB} .
\end{gathered}
$$

Note: This example is the same as that studied in [11] and [12] although the SNR is $10 \mathrm{~dB}$ in [12].

\section{B. Example 2}

$$
\begin{aligned}
f\left(n_{1}, n_{2}\right)= & \sin \left(0.1464 \pi n_{1}+0.2249 \pi n_{2}\right) \\
& +\sin \left(0.2889 \pi n_{1}+0.0778 \pi n_{2}\right) \\
& +g\left(n_{1}, n_{2}\right)
\end{aligned}
$$

for

$$
\begin{aligned}
& 1 \leq n_{1} \leq N_{1} \\
& 1 \leq n_{2} \leq N_{2}
\end{aligned}
$$

and

$$
\begin{gathered}
N_{1}=N_{2}=8 \\
\sigma_{g}^{2}=0.8 \\
\mathrm{SNR}=0 \mathrm{~dB} .
\end{gathered}
$$

In both examples, 90 equispaced projections from $\theta=0^{\circ}$ to $\theta$ $=90^{\circ}$ are taken. The projections are filtered by using a 1-D FIR filter whose frequency response is $|\omega|^{1 / 2}[20]$. The length of the filter chosen is equal to the length of the data. In the first example, for each of the filtered projections a 1-D AR model of order 6 is used. An AR model of order 4 is used in the second example. The Burg algorithm [21] is employed to obtain the AR parameters. The resulting estimate on the polar raster is converted into a cartesian raster using a 2-D firstorder interpolation. This is done for our convenience in plotting the results. The resulting 2-D power spectral estimates are shown in Figs. 4(a) and 5(a). The 2-D periodogram spectral estimates obtained by the same data are shown in Figs. 4(b) and 5(b). Contour plots of only the first quadrant of the PSD estimate are presented in these figures. 


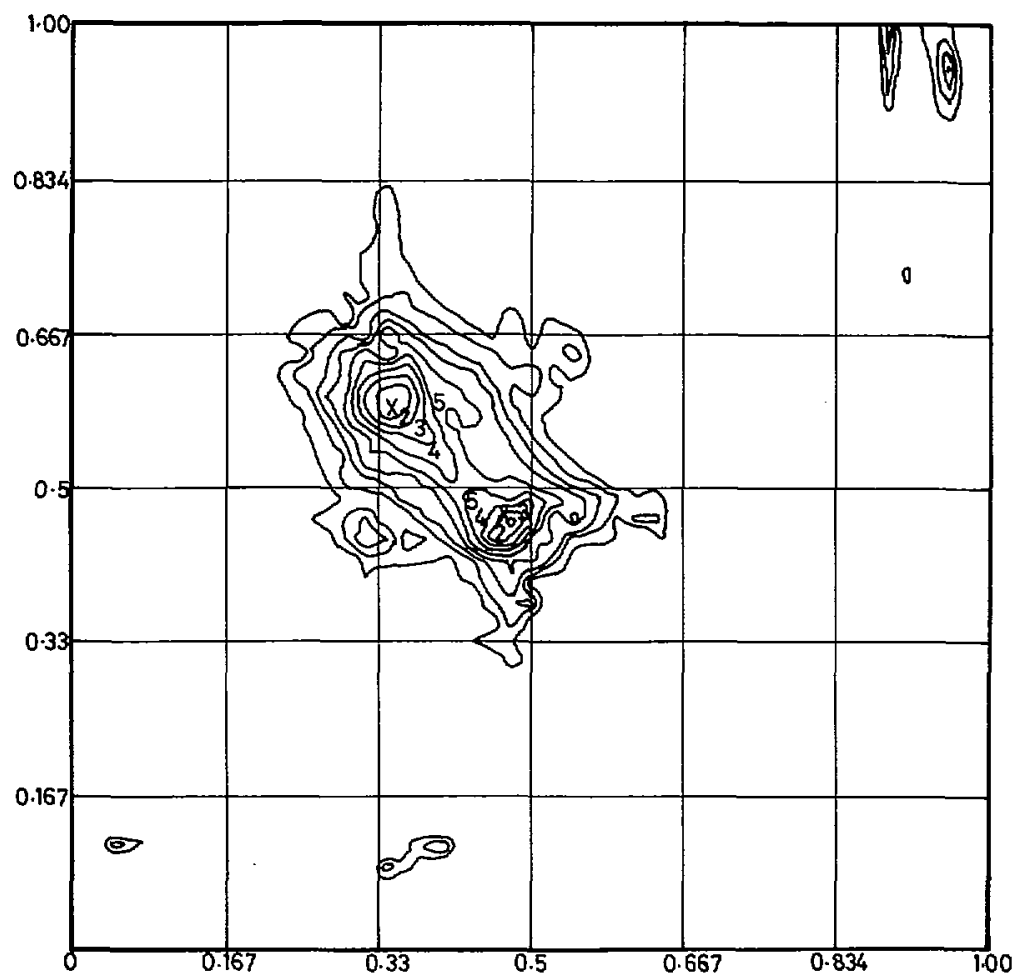

(a)

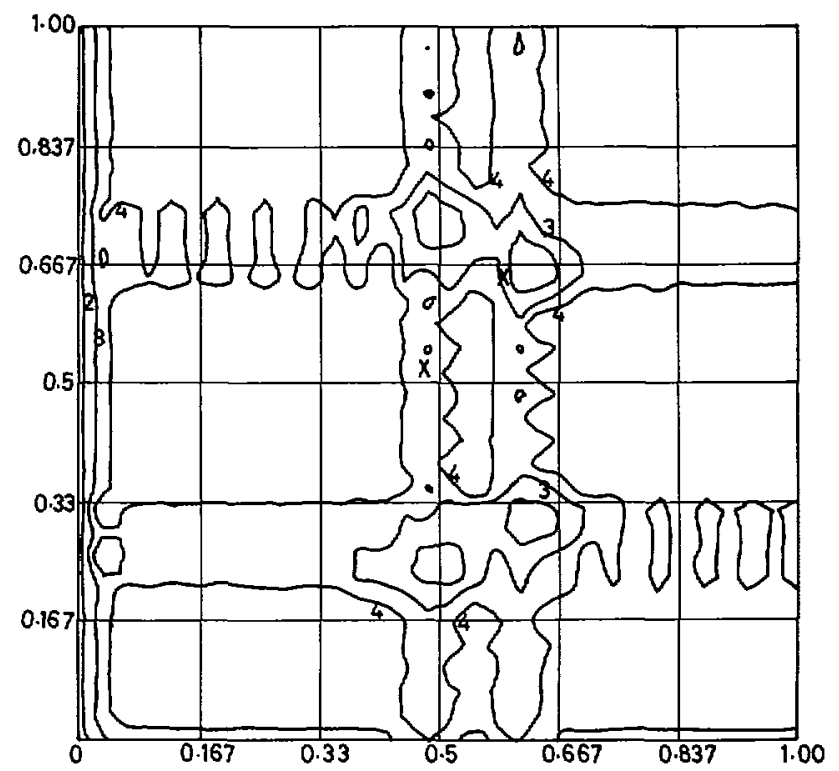

(b)

Fig. 4. Two-dimensional power spectral estimates for Example 1. In all figures " $x$ " indicates the true peak location of the signal sinusoids. The contour levels represent the decibels relative to the maximum value of the estimated spectrum. The contour levels are spaced $-3 \mathrm{~dB}$ apart in 4 (a) and 4(b) and $-10 \mathrm{~dB}$ apart in 4(b) and 5(b). (a) Result of PSD estimate using $\mathrm{AR}(6)$ for 90 equispaced projections. (b) Result of the periodogram estimate.

It is evident from the contour plots that the PSD estimates obtained by the proposed technique have excellent frequency resolution capability. In the first example, the spectral estimates have peaks at the desired frequencies and a smooth spectrum for most other frequencies. The power level midway between the two desired frequency pairs is about $10 \mathrm{~dB}$. This result, which is obtained by using a 1-D AR model, is superior to that obtained in [11], where 2-D quarter-plane AR models are used, in spite of the approximations made. The several false peaks which are very much evident in the results in [11] 


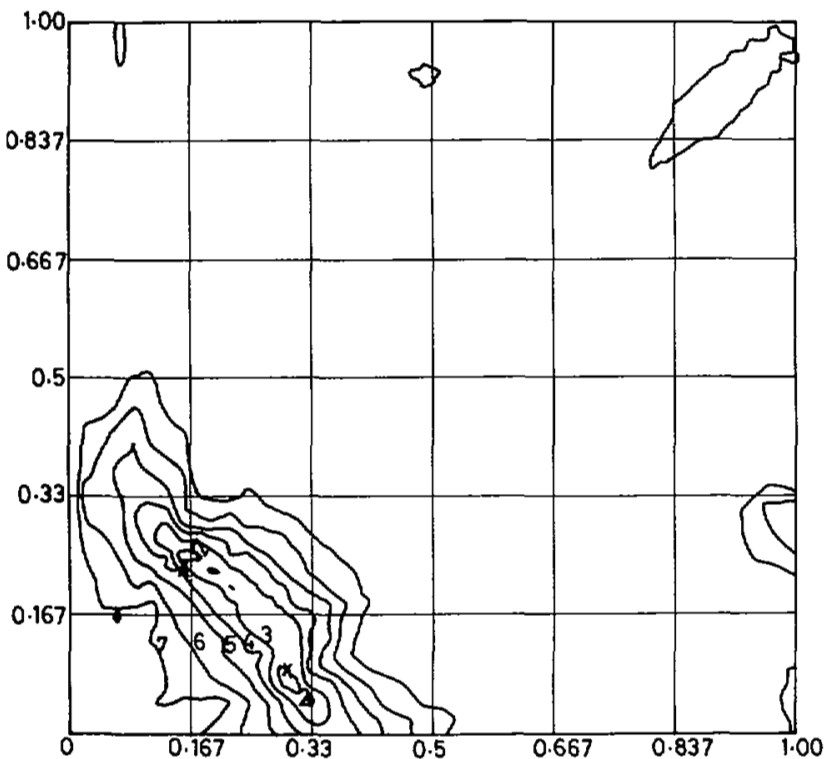

(a)

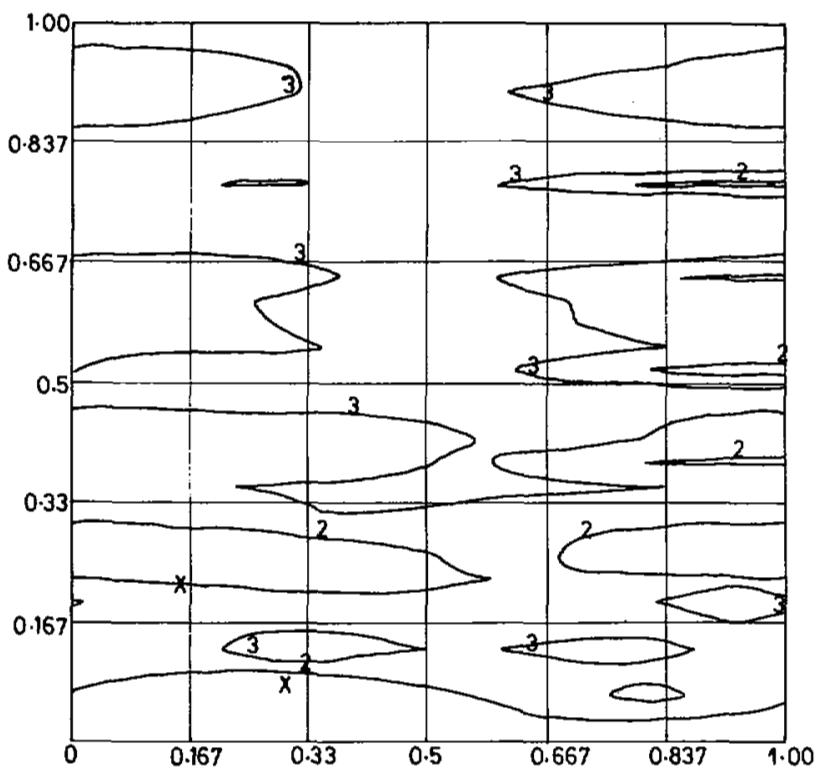

(b)

Fig. 5. Two-dimensional PSD estimates for Example 2. (a) Result of PSD estimate using AR(4) for 90 equispaced projections. (b) Result of the periodogram estimate.

are completely absent in Fig. 4(a). Also this result is in no way inferior to those estimates obtained using 2-D quarter-plane ARMA models in [11] and the 2-D noncausal AR spectral estimate in [12]. In the second example where the observations are known only at $(8 \times 8)$ points, the plot shows that there are a few false peaks. Some of the reasons that could be attributed to this are the use of 1-D AR models and the error due to the interpolation from the polar to Cartesian coordinates. The use of 1-D ARMA models in this case may yield a better estimate. The error due to polar-to-Cartesian conversion can be completely avoided by displaying the results directly in polar form. The periodogram estimates give very broad peaks in the first example and are not able to resolve the two peaks in the second. The well-known random fluctuation behavior which is a typical characteristic of the periodogram can also be seen. These examples demonstrate the performance of the new approach.

\section{Comments}

The approach chosen in this paper in approximating the continuous Radon transform by the "pixel" assumption and the summation operator generally results in attenuation of high-frequency components. The pixel assumption is a fundamental assumption in digital image processing.

An alternative approach is to use the discrete Radon transform (DRT). A major problem in using the DRT is that while deriving the projections, estimation of additional samples by an interpolation process is unavoidable (e.g., see [22]). As the generation of additional data by an interpolation process is equivalent to low-pass filtering of the signal [23], this approach will also result in high-frequency attenuation.

The results obtained in polar coordinates have been displayed in Cartesian coordinates for the sake of our convenience. The error involved in the polar-to-Cartesian conversion need not be considered, as the result can be displayed directly in polar coordinates as well.

\section{CONCLUSION}

A novel approach for the PSD estimation from a finite set of observations of a 2-D SRF has been presented. The 2-D observations are reduced to a set of 1-D sequences by Radon transform. The 1-D sequences are then independently modeled by 1-D LP techniques to obtain slices of 2-D PSD. The various features of this approach have been highlighted. The effectiveness of this technique in resolving two closely spaced sinusoids from noisy measurements is shown through simulation examples. The approximations while handling discrete data have been mentioned and the effect of this on the performance of the method needs to be studied further. Work on the application of this technique to the bearing estimation problem in sonar is currently being undertaken.

An interesting issue arises. It is well known that in the 1-D case the AR spectral estimate is equivalent to the maximum entropy estimate. Since the 1-D AR spectral estimate of each of the filtered projections of the 2-D stationary random field form different slices of the 2-D spectra, it may be interesting to study the duality in the 2-D case along this direction.

\section{ACKNOWLEDGMENT}

The authors thank the anonymous reviewers for their critical comments, which improved the clarity of the paper.

\section{REFERENCES}

[1] D. G. Childers, Ed., Modern Spectral Analysis. New York: IEEE, 1978.

[2] J. H. McClellan, "Multidimensional spectral estimation," Proc. IEEE, vol. 70, pp. 1029-1039, Sept. 1982.

[3] P. L. Jackson, L. S. Joyce, and G. B. Feldkamp, "Application of maximum entropy frequency analysis to synthetic aperture radar," in Proc. RADC Spectrum Estimation Workshop (Rome, NY), May 1978, pp. 217-225. 
[4] T. J. Ulrych and C. J. Walker, "High resolution 2-dimensional power spectral estimation," in Applied Time Series Analysis II, D. F. Findley, Ed. New York: Academic, 1981.

[5] L. S. Joyce, "A separable 2-D autoregressive spectral estimation algorithm," in Proc. ICASSP 79 (Washington, DC), Apr. 1979, pp. $677-680$.

[6] O. L. Frost and T. M. Sullivan, "High resolution two-dimensional spectral analysis," in Proc. ICASSP (Washington, DC), Apr. 1979, pp. $673-676$.

[7] A. K. Jain and S. Ranganath, "Extrapolation algorithms for discrete signals with application in spectral estimation," IEEE Trans. Acoust. Speech, Signal Processing, vol. ASSP-29, pp. 830-845, Aug. 1981.

[8] A. K. Jain, "Advances in mathematical models for image processing," Proc. IEEE, vol. 69, pp. 349-389, May 1981.

[9] L. B. Jackson and H. C. Chien, "Frequency and bearing estimation by two dimensional linear prediction," in Proc. ICASSP 79 (Washington, DC), Apr. 1979, pp. 665-668.

[10] O. L. Frost, "High resolution 2-D spectral analysis at low SNR," in Proc. ICASSP 80 (Denver, CO), Apr. 1980, pp. 580-583

[11] J. A. Cadzow and K. Ogino, "Two-dimensional spectral estimation," IEEE Trans. Acoust, Speech, Signal Processing, vol. ASSP-29, pp. 396-401, June 1981.

[12] G. Sharma and R. Chellappa, "Two-dimensional spectrum estimation using noncausal autoregressive models," IEEE Trans. Inform. Theory, vol. IT-32, pp. 268-275, Mar. 1986.

[13] J. V. Pendrell and D. E. Smylie, "The maximum entropy principle in two-dimensional spectral analysis," Astron. Astrophys., vol. 112, pp. 181-189, 1982.

[14] D. Tjostheim, "Autoregressive modelling and spectral analysis of array data in the plane," IEEE Trans. Geosci. Remote Sensing, vol. GE-19, pp. 15-24, Jan. 1981.

[15] T. L. Marzetta, "Two-dimensional linear prediction: Autocorrelation arrays, minimum-phase prediction error filters, and reflection coefficient arrays," IEEE Trans. Acoust., Speech, Signal Processing, vol. ASSP-28, pp. 725-733, Dec. 1980.

[16] D. E. Dudgeon and R. M. Mersereau, Multi-dimensional Signal Processing. Englewood Cliffs, NJ: Prentice-Hall, 1984.

[17] J. Radon, "Uber die Bestimmung von Funktionen durch ihre Integralwerte langs gewisser Manningfaitigkeiten," Bwerichte Sachsische Akademie der Wissenschafter, Leipzig, Math-Phys, KL. 69, pp. 262$279,1917$.

[18] S. R. Deans, The Radon Transform and Some of Its Applications. New York: Wiley, 1983.

[19] A. K. Jain and S. Ansari, "Radon transform theory for random fields and optimum image reconstruction from noisy projections," in Proc. ICASSP 84 (California), Mar. 1984.

[20] H. H. Barrett and W. Swindell, Radiological Imaging, vol. $\amalg$. New York: Academic, 1981.

[21] J. P. Burg, "A new analysis technique for time series data," presented at NATO Advanced Study Inst. Signal Processing with Emphasis on Underwater Acoustics, Enschede, Netherlands, 1968.
[22] D. J. Scheibner and T. W. Parks, "Slowness aliasing in the discrete Radon transform: A multirate approach to beam forming," IEEE Trans. Acoust., Speech, Signal Processing, vol. ASSP-32, pp. 11601165, Dec. 1984.

[23] R. E. Crochiere and L. R. Rabiner, Multirate Digital Signal Processing. Englewood Cliffs, NJ: Prentice-Hall, 1983.

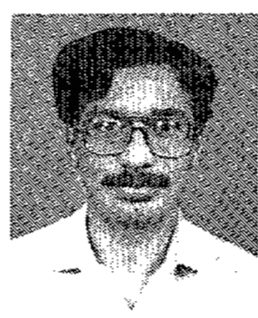

N. Srinivasa (S'83) was born in Bangalore, India, on November 26, 1961. He received the B.E. degree in electronics and communication engineering from Mysore University in 1983. He is currently completing the requirements for the $\mathrm{Ph} . \mathrm{D}$. degree in electrical engineering at the Indian Institute of Science, Bangalore.

His research interests include digital signal processing with applications to computerized tomography, adaptive signal processing, spectral estimation, image processing, and VLSI architectures.

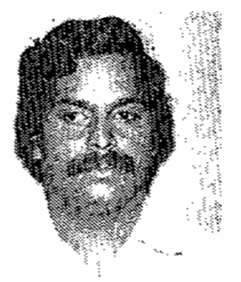

K. R. Ramakrishnan (S'84-M'84) received the B.E., M.E., and Ph.D. degrees in electrical engineering from the Indian Institute of Science, Bangalore, in 1974, 1976, and 1983, respectively.

Currently, he is Assistant Professor in the Department of Electrical Engineering at the Indian Institute of Science. His current interests are computer vision and image processing, artificial intelligence, and signal processing.

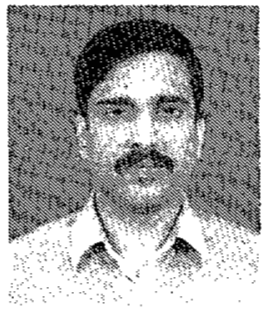

K. Rajgopal (S'84-M'84) was born in Vizianagaram, India, on May 9, 1953. He received the B.E. degree in electronics and communication from Nagpur University in 1974 and the Ph.D. degree in electrical engineering from the Indian Institute of Science, Bangalore, in 1975.

He served as Scientific Assistant at the Indian Institute of Science from 1977 to 1983, as Scientific Officer from 1983 to 1985 , and as Lecturer from 1985 to the present. His current interests are in the areas of digital signal processing, computer aided tomography, sonar signal processing, and active and digital filters. Dr. Rajgopal is a member of IETE India. 